\title{
Polietileno de Alta Densidade Tenacificado com Elastômero Metalocênico: 1. Propriedades Mecânicas e Características Morfológicas
}

\author{
Maria José 0. C. Guimarães \\ Departamento de Processos Orgânicos, EQ/UFRJ
}

\author{
Marisa C. G. Rocha \\ Instituto Politécnico da UERJ
}

Fernanda M. B. Coutinho

IMA/UFRJ e Departamento de Processos Químicos, IQ/UERJ

\begin{abstract}
Resumo: Neste trabalho foram estudadas as propriedades mecânicas e morfológicas de polietileno de alta densidade (HDPE) tenacificado com dois tipos de elastômeros metalocênicos à base de etileno/1- octeno (EOC). Esses elastômeros são polímeros comerciais com diferenças quanto ao peso molecular, índice de fluidez e índice Dow de reologia (DRI). Misturas físicas de HDPE e EOC foram processadas em extrusora monorosca Wortex $(\mathrm{L} / \mathrm{D}=32)$, à $230^{\circ} \mathrm{C}$ e $50 \mathrm{rpm}$, utilizando percentagem mássica do EOC de $5 \%$ a $80 \%$. Foi observado um efeito sinergístico nas propriedades tênseis e características de supertenacificação para materiais contendo proporções do EOC maiores do que 5\%. Cavitação, deformação plástica e cavitação fibrilada foram observados nos processos de deformação. Materiais contendo até 50\% do EOC apresentaram morfologias dispersas com domínios elastoméricos esféricos, distribuídos uniformemente e com tamanho médio de partícula na faixa de 0,30 a $0,45 \mu \mathrm{m}$. A tenacificação de HDPE com os elastômeros etilênicos produziu materiais com boas propriedades e compatibilização tecnológica devido à existência de baixa tensão interfacial entre esses polímeros.
\end{abstract}

Palavras-chave: HDPE, elastômeros metalocênicos, tenacificação, morfologia.

\section{Rubber Toughened High Density Polyethylene: 1. Mechanical Properties and Morphological Characteristics}

Abstract: The mechanical and morphological properties of high density polyethylene (HDPE) toughened with two different grades of metallocene elastomers based on ethylene/1- octene (EOC) were studied. These elastomers were commercial polymers differing in molecular weight, melt flow index and Dow rheology index (DRI). Blends were processed in a Wortex single screw extruder $(\mathrm{L} / \mathrm{D}=32)$, at $230^{\circ} \mathrm{C}$ and $50 \mathrm{rpm}$, using mass fraction weight percent of EOC in the range from $5 \%$ to $80 \%$. A synergistic effect on the tensile properties and supertough behavior for blends with EOC concentrations higher than 5\% was observed. Cavitation, plastic deformation and fibrillized cavitation were observed in the deformation processes. Materials containing up to $50 \%$ of EOC exhibited dispersed morphologies with EOC spherical domains uniformly distributed with particle size in the range from 0.30 to $0.45 \mu \mathrm{m}$. The toughening of HDPE with ethylenic elastomers produced materials with good properties and technological compatibilization.

Keywords: HDPE, metallocene elastomers, toughening, morphology.

Autor para correspondência: Fernanda M. B. Coutinho, UERJ / IQ e UFRJ / IMA, CP:68525, CEP: 21945-970, Rio de Janeiro, RJ. E-mail: fern@ima.ufr.br 


\section{Introdução}

Com o desenvolvimento de sistemas catalíticos baseados em metalocenos, polímeros etilênicos com estreito índice de polidispersão e distribuição homogênea de comonômeros alfa-olefínicos na estrutura macromolecular, são hoje industrialmente disponíveis. Essa nova classe de polietilenos modificados pela natureza de suas ramificações de cadeia longa e pelo arranjo das ramificações de cadeia curta na macromolécula, quando em misturas com poliolefinas (PE e PP), permitem a obtenção de materiais de fácil processamento e com propriedades específicas para aplicação como materiais de engenharia ${ }^{[1-7]}$.

Polietileno de alta densidade apresenta boas propriedades de resistência à tração e ductilidade a temperatura ambiente, entretanto sob condições severas de alta pressão, baixa temperatura e altas velocidades de deformação, torna-se quebradiço. Tubulações feitas de polietileno quando submetidas a altas pressões e/ou baixas temperaturas apresentam o fenômeno da propagação rápida da trinca da fratura frágil. $\mathrm{O}$ HDPE para ser utilizado nessas condições necessita ser tenacificado. Isso pode ser feito pela incorporação de material elastomérico utilizando processo de mistura no estado fundido ${ }^{[8,9]}$.

A tenacidade é uma das características mais importantes que determina se um dado polímero pode ser usado ou não como material de engenharia ${ }^{[10]}$. Nas misturas de termoplásticos reforçados com elastômeros, quando se aplica uma tensão, as partículas elastoméricas dispersas concentram ou absorvem essa tensão, provocando uma alteração do estado de tensão da fase matricial, e uma intensa deformação plástica. A absorção-dissipação de energia pelas partículas dispersas se processa por diferentes mecanismos, tais como, cavitação (ruptura), deformação plástica (escoamento), ou por duplo processo de cavitação-deformação ${ }^{[10-13]}$.

A eficiência da tenacificação depende em geral do tipo específico do modificador empregado, e de alguns parâmetros a este relacionado como fração volumétrica, tamanho e distribuição de tamanhos de partícula, temperatura de transição vítrea e afinidade química com a matriz ou nível de adesão interfacial $^{[10,11,13-15]}$. A tenacificação, ou mudança de comportamento de frágil para dúctil, sofre também influência das condições de teste (velocidade e temperatura), e das propriedades mecânicas da matriz e das partículas de modificador ${ }^{[10,16-18]}$.
A tenacificação de polímeros cristalizáveis, tais como, poliamidas e as poliolefinas, PE e PP, é ainda um assunto controverso e de estudo relativamente recente. Alguns autores consideram que o aumento da resistência ao impacto desses materiais está também relacionado à formação de camadas cristalinas interconectadas em torno das partículas de modificador. Assim, segundo essa teoria, existe uma espessura crítica da camada, específica para cada tipo de matriz, abaixo da qual uma tenacificação máxima é observada $^{[9,19]}$.

Neste trabalho foram estudadas as propriedades mecânicas e morfológicas de polietileno de alta densidade tenacificado com dois tipos de elastômeros metalocênicos à base de etileno e 1-octeno comerciais. O uso dos elastômeros olefínicos metalocênicos para a tenacificação de poliolefinas é conveniente devido à similaridade química entre os componentes da mistura, favorecerendo assim uma melhor adesão interfacial. Além disso, esses elastômeros apresentam maior estabilidade térmica; são mais facilmente estabilizados com aditivos contra radiação UV, apresentando ainda maior eficiência de reticulação sob a ação de peróxidos, que os tradicionais elastômeros de etileno/propileno (EPR) e etileno/propileno/dieno (EPDM) ${ }^{[11,20]}$.

\section{Experimental}

\section{Materiais e Processamento}

A Tabela 1 apresenta as especificações dos polímeros utilizados neste trabalho. A incorporação de material elastomérico à matriz do HDPE foi feita em extrusora monorosca Wortex específica para poliolefinas $(\mathrm{L} / \mathrm{D}=32)$, à $230^{\circ} \mathrm{C}$ e $50 \mathrm{rpm}$. Foram utilizadas concentrações mássicas dos elastômeros EOC de $5 \%$ a $80 \%$ e cerca de $0,1 \%$ de Irganox como agente antioxidante.

\section{Propriedades Mecânicas}

As propriedades mecânicas foram medidas em equipamento Instron 4204 a temperatura ambiente $\left(25^{\circ} \mathrm{C}\right.$ e $55 \%$ de umidade relativa), com velocidade de estiramento $500 \mathrm{~mm} / \mathrm{min}$, de acordo com a norma ASTM D882, utilizando cerca de 10 corpos-de-prova obtidos por processo de extrusão. A resistência ao impacto foi determinada em equipamento Ceast utilizando o método de Izod com entalhe segundo a norma ASTM D256 e cerca de 6 corpos de prova obtidos 
Tabela 1. Características dos polímeros

\begin{tabular}{|c|c|c|c|c|}
\hline Especificações & & HDPE & EOC10 & EOC20 \\
\hline Produtor & & Polialden & Dow Chemical & Dow Chemical \\
\hline Nome comercial & & ET010M & Engage 8100 & Engage 8200 \\
\hline \multirow[t]{2}{*}{ Índice de fluidez (g/10min) } & & 0,93 & 1,0 & 5,0 \\
\hline & $\mathrm{Mn}$ & 74.420 & 155.600 & 93.870 \\
\hline \multirow[t]{2}{*}{ Peso Molecular } & Mw & 285.700 & 323.000 & 196.600 \\
\hline & $\mathrm{Mw} / \mathrm{Mn}$ & 3,8 & 2,07 & 2,09 \\
\hline Percentagem de octeno & & - & 24 & 24 \\
\hline Densidade $\left(\mathrm{g} / \mathrm{cm}^{3}\right)$ & & 0,960 & 0,870 & 0,870 \\
\hline Índice Dow de Reologia (DRI) ${ }^{\mathrm{a}}$ & & - & 2,0 & 0,5 \\
\hline
\end{tabular}

(a) Concentração de ramificações de cadeia longa

por processo de injeção. As superfícies de fratura após os testes de impacto foram cobertas com uma fina camada de liga ouro/paládio e observadas em microscópio eletrônico de varredura.

\section{Caracterização Morfológica}

A morfologia do sistema foi examinada em microscópio eletrônico de varredura Zeiss modelo DSM 960. As amostras foram fraturadas em nitrogênio líquido e a fase elastomérica extraída com xileno à $60^{\circ} \mathrm{C}$ por 30 segundos. A superfície tratada foi então coberta com uma fina camada de liga ouro/paládio e analisada sob uma voltagem de $15 \mathrm{kV}$.

\section{Resultados e discussão}

A Figura 1 apresenta as curvas de tensão de ruptura e alongamento de ruptura em função da percentagem mássica do EOC. Um efeito sinergístico na tensão e alongamento de ruptura foi obtido pela incorporação do EOC à matriz do HDPE. Esse efeito é mais acentuado para percentagens de EOC na faixa de $30 \%$ a $50 \%$. Um comportamento similar foi também observado por outros pesquisadores em sistemas de HDPE modificado com copolímeros etilênicos elastoméricos e termoplásticos $^{[22,23]}$. Nos ensaios de tração o HDPE puro e tenacificado tornaram-se esbranquiçados na região de estiramento. $\mathrm{O}$ fenômeno de embranquecimento sob tensão (stress whitening) desses materiais, durante o processo de deformação, é geralmente a manifestação de um processo de cavitação em escala fina e, sugere que tanto o HDPE quanto o EOC podem cavitar, produzindo microcavidades com conseqüente modificação do índice de refração do material durante a deformação plástica nas condições de teste utilizadas ${ }^{[9,24]}$.

O módulo de elasticidade em tração, ou módulo de Young, para misturas poliméricas é fortemente dependente da composição, e principalmente, da morfologia do sistema ${ }^{[25]}$. Várias equações podem ser encontradas na literatura para predizer a dependência do módulo em função da composição da mistura. Essas equações são fundamentadas principalmente na lei da aditividade de misturas. Os módulos são geralmente preditos utilizando as equações 1-1 e 1-2 para uma ampla faixa de padrões morfológicos ${ }^{[25]}$. Para misturas com morfologia especificamente co-contínua, Willemse e colaboradores desenvolveram a equação 1-3 $3^{[26]}$.

$$
\begin{aligned}
& \mathrm{M}=\mathrm{M}_{1} \phi_{1}+\mathrm{M}_{2} \phi_{2} \\
& \frac{1}{\mathrm{M}}=\frac{\phi_{1}}{\mathrm{M}_{1}}+\frac{\phi_{2}}{\mathrm{M}_{2}} \\
& \mathrm{M}_{\mathrm{M}}^{1 / 5}=\mathrm{M}_{1}^{1 / 5} \phi_{1}+\mathrm{M}_{2}^{1 / 5} \phi_{2}
\end{aligned}
$$

Onde:

M é o módulo da mistura; $\mathrm{M}_{1}, \mathrm{M}_{2}$ e $\phi_{1}$ e $\phi_{2}$ são respectivamente os módulos e as frações volumétricas dos componentes. 


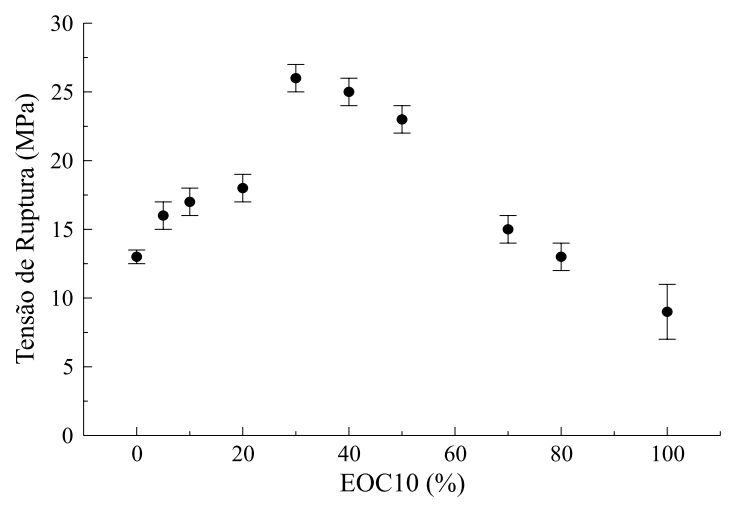

(a)

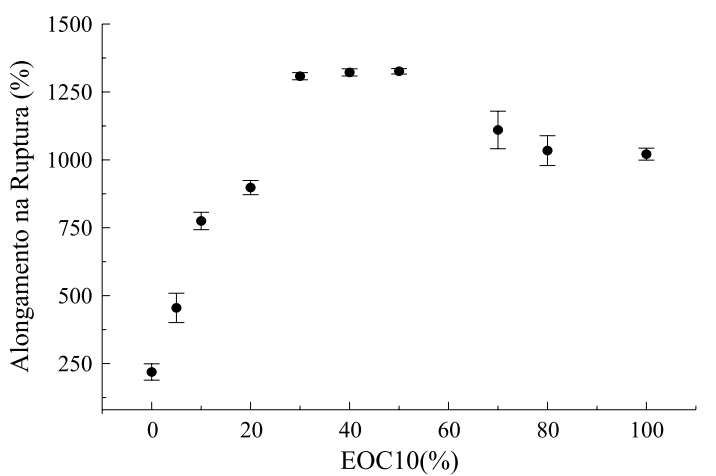

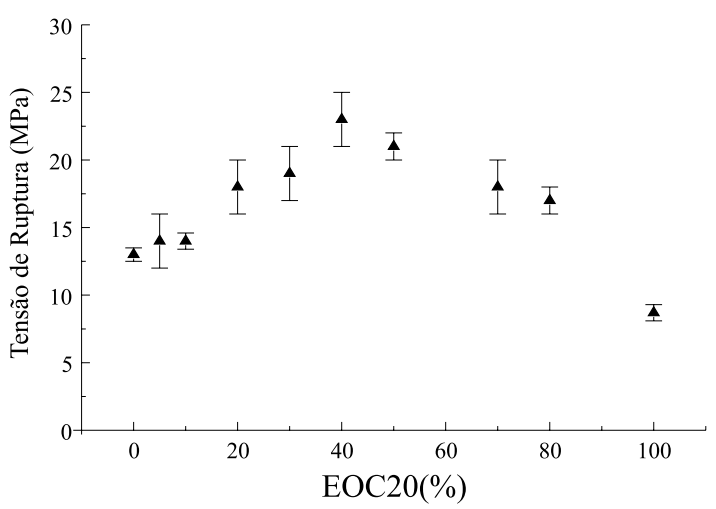

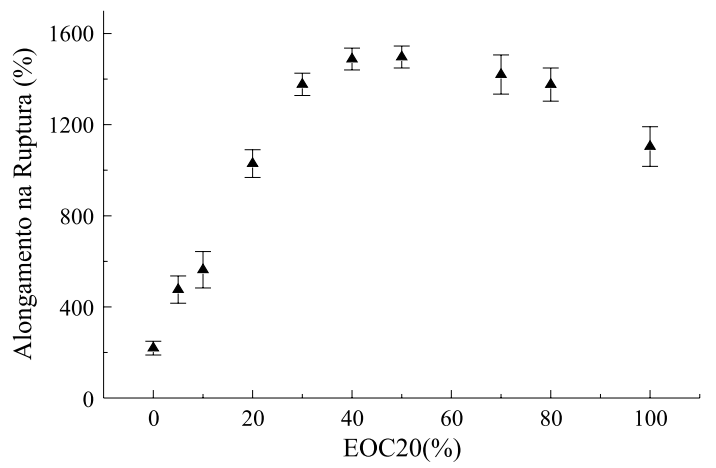

(b)

Figura 1. Propriedades tênseis para os sistemas HDPE/EOC: (a) tensão de ruptura, (b) alongamento de ruptura

A Figura 2 apresenta os valores experimentais e os valores preditos para o módulo de Young utilizando as equações 1-1, 1-2 e 1-3.

$\mathrm{O}$ módulo de Young está associado à rigidez do material e tende a decrescer com o aumento da concentração do componente elastomérico. Para as misturas HDPE/EOC com percentagens volumétricas de até 20\% de EOC, os valores dos módulos experimentais tendem a ser praticamente constantes e estão mais próximos dos valores preditos pela equação 1-1. Para percentagens maiores do que $20 \%$, os módulos decrescem continuamente e tendem a se aproximar dos valores preditos pela

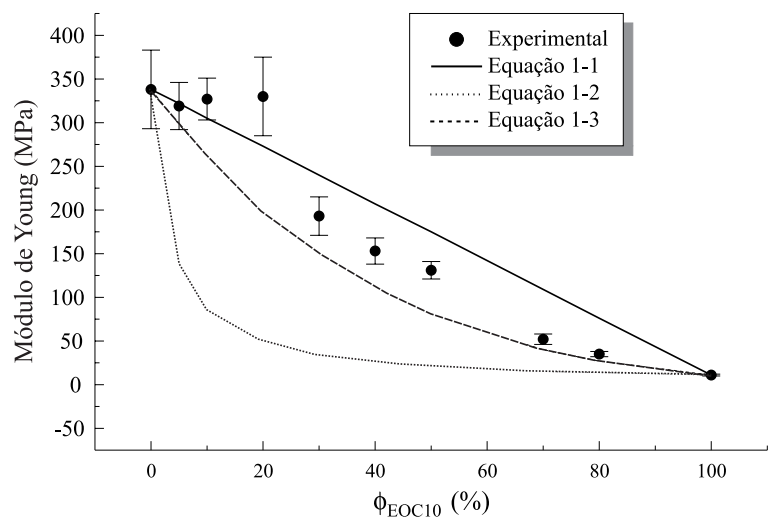

equação 1-3. A equação 1-3 adequa-se bem aos sistemas do tipo elastômero reforçado com termoplástico, ou seja, para altíssimas concentrações de EOC. Em termos de padrão morfológico, os materiais com altas percentagens de EOC tendem a apresentar uma morfologia mais próxima do tipo co-contínua que dispersa, como também predita pelo modelo ${ }^{[16]}$.

A tenacificação de HDPE com os elastômeros metalocênicos EOC modificou significativamente as suas propriedades. Essas modificações puderam ser quantificadas por meio de ensaios de tensão-deformação. O processo de tenacificação provocou:

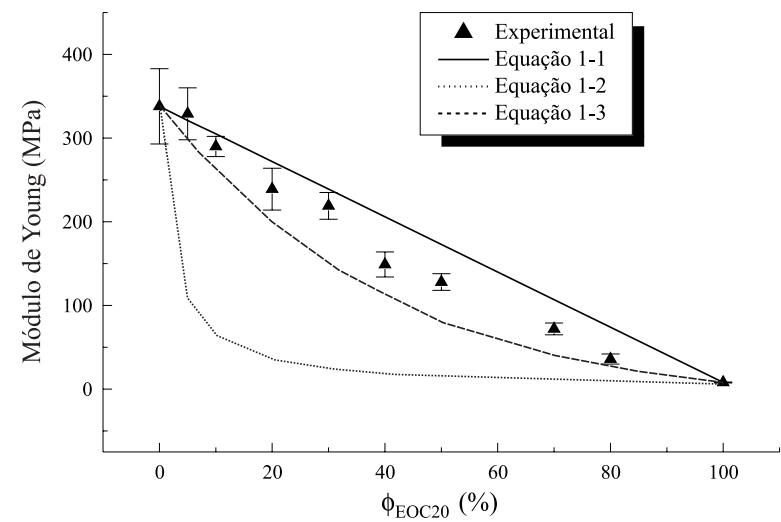

Figura 2. Variação do módulo de Young com a composição para os sistemas HDPE/EOC 
Tabela 2. Resistência ao impacto do HDPE tenacificado a $25^{\circ} \mathrm{C}$

\begin{tabular}{ccc}
\hline \multirow{2}{*}{ EOC $(\%)$} & \multicolumn{2}{c}{$\mathbf{R I}(\mathbf{J} / \mathbf{m})$} \\
\cline { 2 - 3 } & HDPE/EOC10 & HDPE/EOC20 \\
\hline 0 & $297 \pm 20$ & $297 \pm 20$ \\
5 & $457 \pm 21$ & $581 \pm 40$ \\
$>10$ & $\mathrm{a}$ & $\mathrm{a}$ \\
\hline
\end{tabular}

(a) Não fraturou sob as condições de teste

a) Modificação do pico de escoamento sob tensão;

b) Aumento do alongamento de ruptura;

c) Embranquecimento do material (stress whitening) durante o alongamento;

d) Aumento da energia de ruptura;

e) Diminuição do módulo elástico para concentrações de EOC maiores do que $20 \%$.

Os valores de resistência ao impacto do HDPE tenacificado são mostrados na Tabela 2. A tenacificação do HDPE com 5\% dos elastômeros EOC10 e EOC20, resultou respectivamente em um aumento de $53 \%$ e $96 \%$ da resistência ao impacto em comparação com o HDPE puro. Para concentrações de EOC maiores do que 5\%, o HDPE apresentou comportamento de supertenacificação e não fraturou sob as condições de teste usadas. Todas as amostras tornaram-se esbranquiçadas na região próxima da fratura. O nível de embranquecimento aumentou com o aumento da percentagem mássica de EOC no material. Comportamento semelhante foi também observado por Bartczak et al. em sistemas de HDPE/elastômeros termoplásticos ${ }^{[9]}$.

As Figuras 3 e 4 apresentam as micrografias de microscopia eletrônica de varredura das superfícies fraturadas dos corpos de prova após a realização do teste de impacto. Como pode ser observado, essas superfícies exibem processos de cavitação e/ou deformação plástica (escoamento). O processo de cavitação parece ter sido maior para a tenacificação com o EOC10, onde se observa o fenômeno da propagação rápida da trinca da fratura frágil em maior extensão do que com o elastômero EOC20. Esses resultados corroboram os resultados experimentais compilados na Tabela 2. Também podem ser observadas inúmeras bandas de cisalhamento e estruturas fibriladas, devido à intensa deformação plástica das fases matricial e modificadora elastomérica. O processo de deformação plástica parece ter sido mais intenso para o siste-



(a)

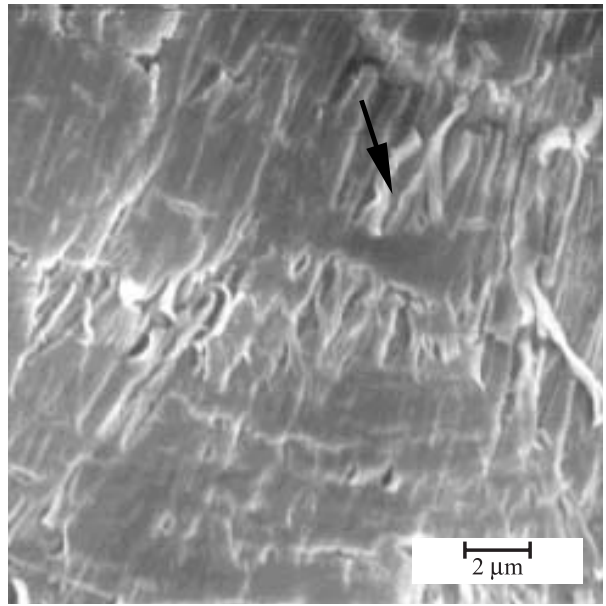

(b)

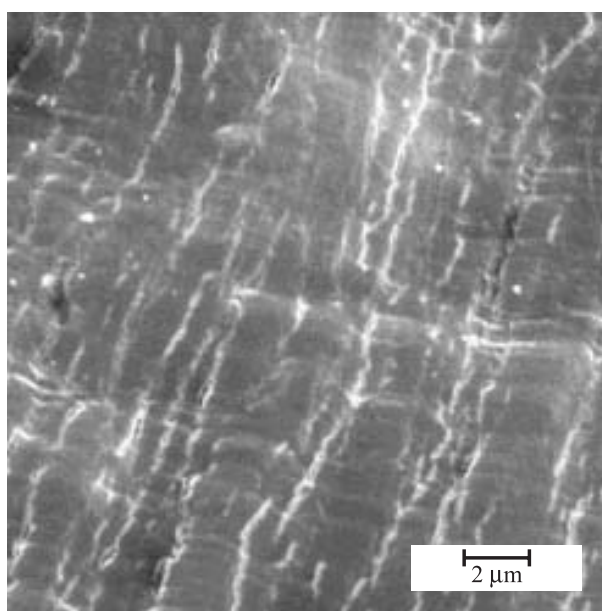

(c)

Figura 3. Superfícies de fratura dos sistemas HDPE/EOC10: (a) a seta indica a propagação da trinca da fratura frágil; (b) e (c) processos de cavitação e/ou deformação (escoamento).

ma tenacificado com o elastômero EOC20 de menor peso molecular e menor concentração de ramificações de cadeia longa, isto é, menor DRI. 


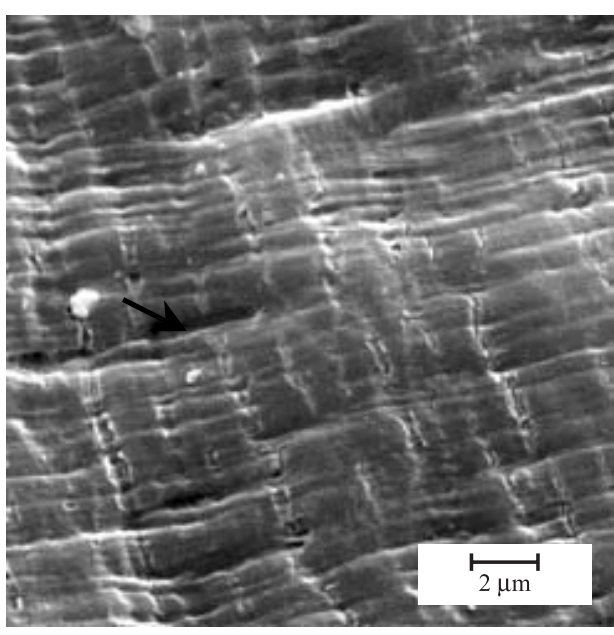

(a)

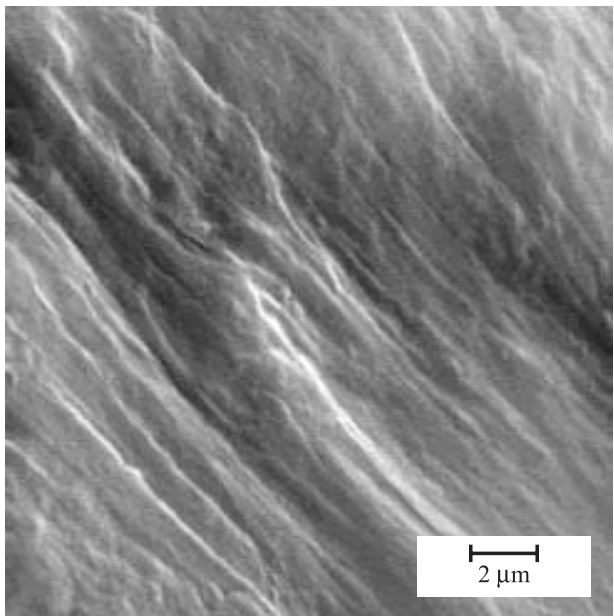

(b)

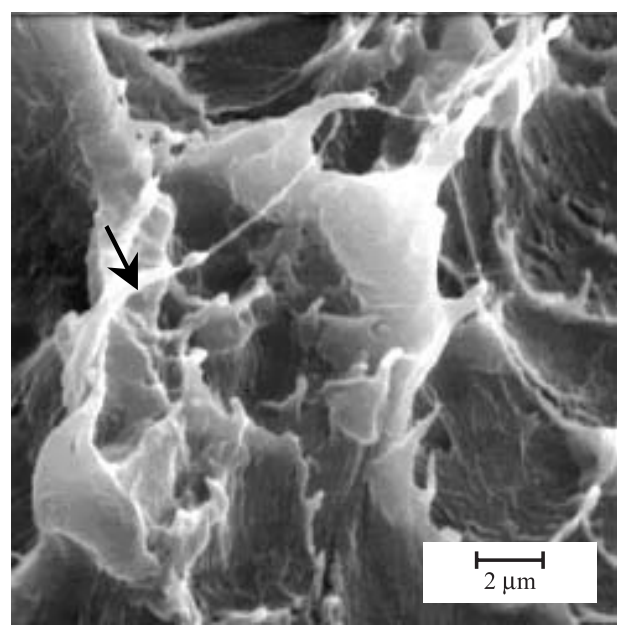

(c)

Figura 4. Superfícies de fratura dos sistemas HDPE/EOC20: (a) cavitação; (b) e (c) processos de deformação (escoamento)

As boas propriedades mecânicas apresentadas pelos sistemas HDPE/EOC, se devem possivelmente

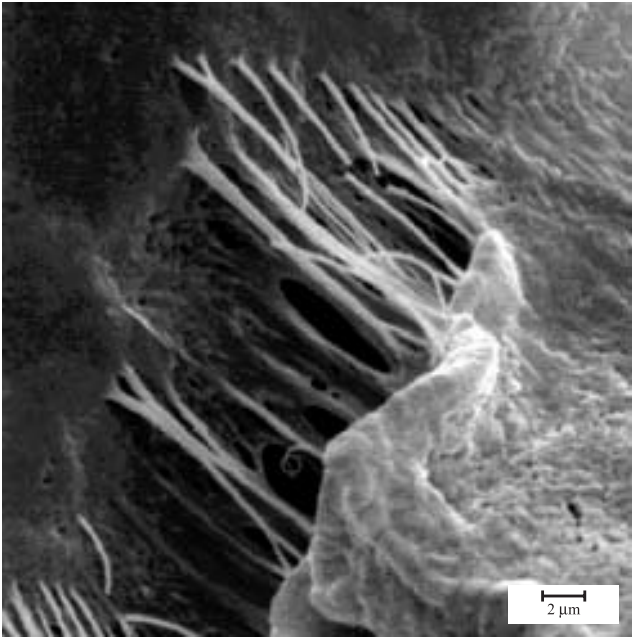

Figura 5. Superfície de fratura criogênica do HDPE tenacificado com cavitação fibrilada

a uma boa adesão entre as fases. Este fato pôde ser confirmado por meio de análise da superfície da fratura criogênica do sistema HDPE/EOC10 contendo $50 \%$ de elastômero (Figura 5). Uma estrutura tenaz com processo de cavitação fibrilada é formada, indicando mais uma vez uma baixa tensão interfacial.

\section{Características Morfológicas}

As variações morfológicas obtidas em função da percentagem mássica dos componentes da mistura, são mostradas nas Figuras 6 e 7. Os dois tipos de sistemas HDPE/EOC apresentam um mesmo padrão morfológico, com domínios esféricos de EOC dispersos em uma matriz de HDPE, quando a percentagem mássica dos elastômeros é de até 40-50\%. Para percentagens mássicas maiores do que $50 \%$, os materiais passam por um processo gradual de inversão de fases com modificação da morfologia que passa a exibir domínios estratificados de HDPE em uma matriz de EOC. Os sistemas HDPE/EOC20 para percentagens mássicas do EOC20 de 80\%, apresentam um padrão morfológico um pouco diferente do sistema HDPE/EOC10, com domínios do HDPE estratificados e já com tendência a dispersão na matriz do EOC20.

O tipo específico de padrão morfológico obtido em um dado sistema polimérico está diretamente relacionado às condições de processamento, composição e principalmente às propriedades reológicas dos componentes. A estabilidade morfológica é função da tensão interfacial entre as fases e da termodinâmica do sistema ${ }^{[27,28]}$. 


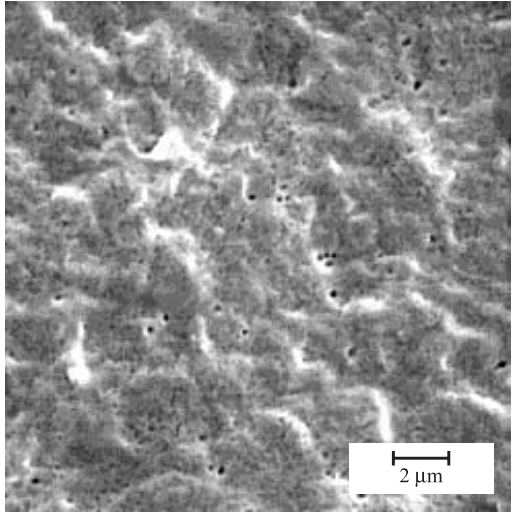

(a)

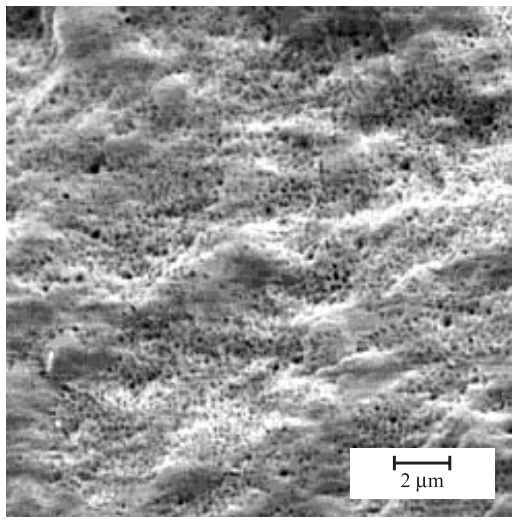

(c)

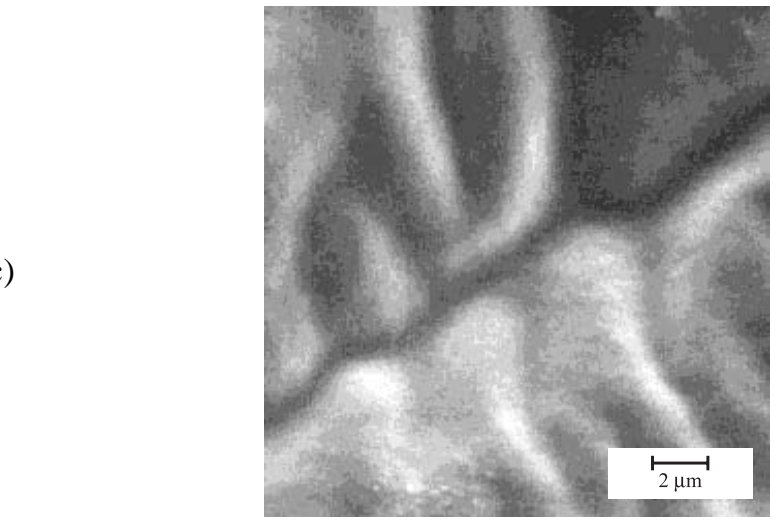

Figura 6. Micrografias dos sistemas HDPE/EOC10; (a) 10\% de EOC10, (b) $20 \%$ de EOC10, (c) $40 \%$ de EOC 10 , (d) $80 \%$ de EOC10



(a)
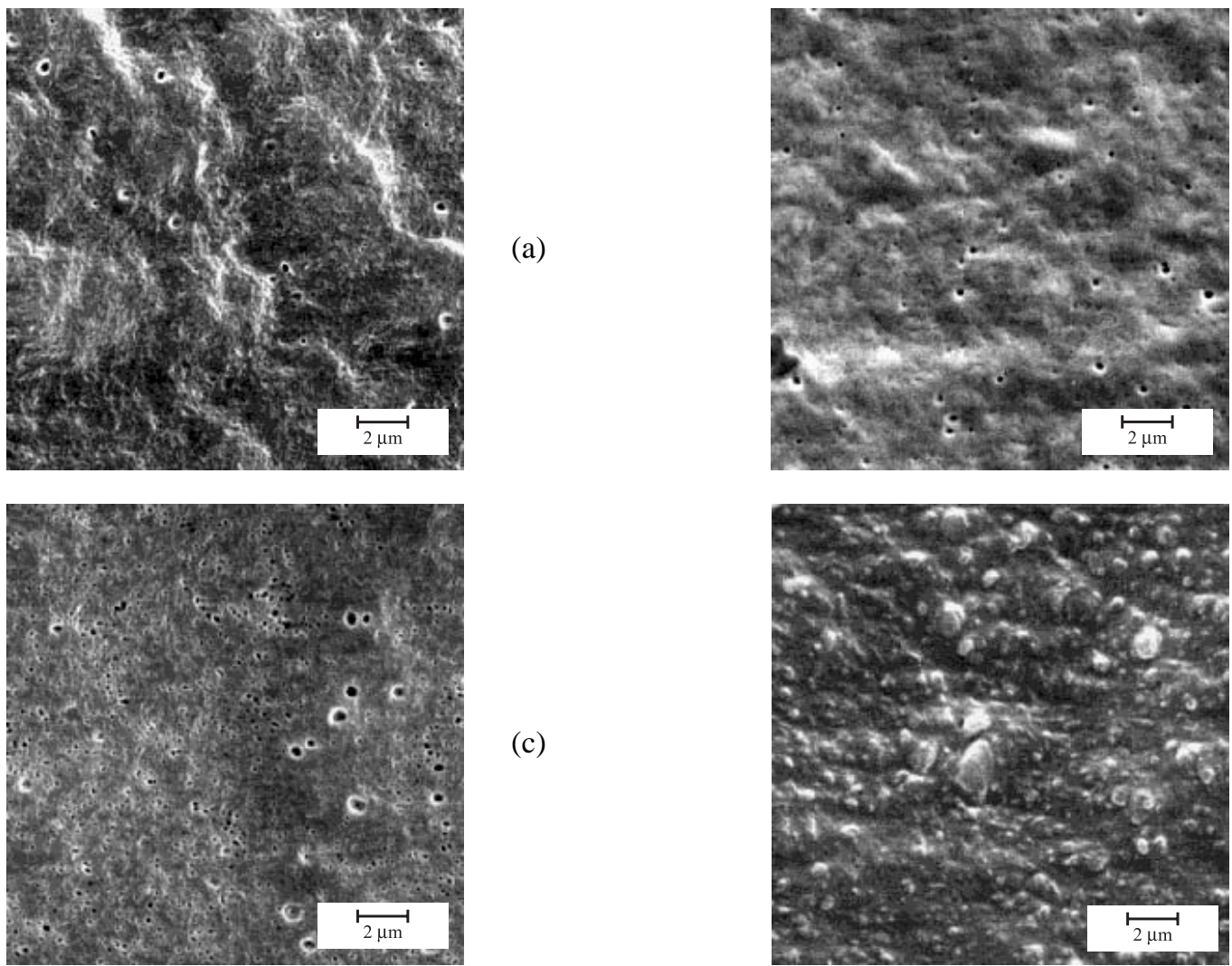

(c)

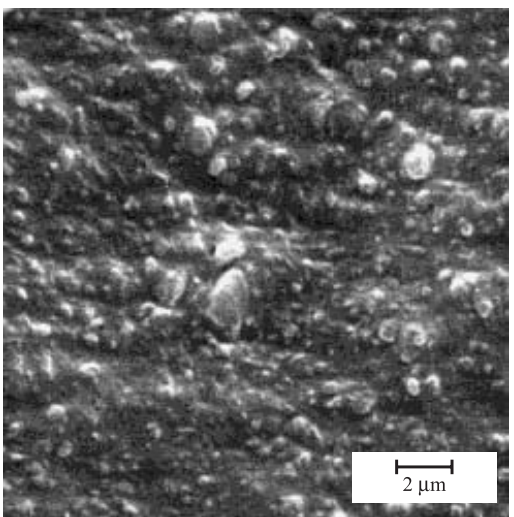

(d)

Figura 7. Micrografias dos sistemas HDPE/EOC20: (a) 10\% de EOC20, (b) $20 \%$ de EOC20, (c) $40 \%$ de EOC20, (d) $80 \%$ de EOC20 
Tabela 3. Diâmetro médio de partícula (D) para os sistemas HDPE/EOC ${ }^{(a)}$

\begin{tabular}{ccc}
\hline \multirow{2}{*}{ EOC $(\%)$} & \multicolumn{2}{c}{$\mathbf{D}(\mu \mathrm{m})$} \\
\cline { 2 - 3 } & HDPE/EOC10 & HDPE/EOC20 \\
\hline 10 & $0,320 \pm 0,16$ & $0,344 \pm 0,18$ \\
20 & $0,326 \pm 0,16$ & $0,350 \pm 0,14$ \\
40 & $0,400 \pm 0,17$ & $0,440 \pm 0,18$ \\
\hline
\end{tabular}

(a) Valores determinados por analisador de imagem

A Tabela 3 fornece o diâmetro médio das partículas (D) para os dois tipos de sistemas HDPE/EOC. Os diâmetros das partículas dispersas de elastômeros apresentam valores próximos na faixa de 0,30 a 0,40 $\mu \mathrm{m}$. Embora havendo uma variação grande de percentagem mássica da fase dispersa, a variação dos valores dos diâmetros das partículas não foi tão acentuada. Este resultado está em concordância com os nossos resultados prévios de análise reológica e corroboram o fato de que os sistemas HDPE/EOC apresentam baixa tensão interfacial tanto no estado fundido quanto no estado sólido ${ }^{[29]}$.

\section{Conclusões}

A tenacificação de HDPE com concentrações específicas dos elastômeros metalocênicos produz materiais com boas propriedades mecânicas para aplicações como materiais de engenharia. Embora os elastômeros apresentem diferenças quanto ao peso molecular e concentração de ramificações de cadeia longa, o comportamento sob tensão-deformação desses sistemas não apresentou diferenças marcantes. Um efeito sinergístico na tensão de ruptura e alongamento de ruptura foi observado indicando a existência de uma boa adesão interfacial. A resistência ao impacto do HDPE foi acentuadamente aumentada de $53 \%$ e $96 \%$ quando foram utilizadas cerca de 5\% de EOC 10 e EOC20 respectivamente no processo de tenacificação. Materiais com características de supertenacificação foram obtidos para concentrações de elastômeros maiores do que 5\%. Cavitação, deformação plástica e cavitação fibrilada foram os processos observados na tenacificação. Quanto menor o peso molecular e a concentração de ramificações de cadeia longa do elastômero mais eficiente foi o processo de tenacificação . O diâmetro médio das partículas de EOC dispersas ficou na faixa de 0,30 a $0,45 \mathrm{~mm}$ e foi pouco influenciado pela composição indicando boa compatibilidade para esses sistemas.

\section{Agradecimentos}

Os autores agradecem aos órgãos de fomento CNPq, FAPERJ, PADCT/CNPq e PRONEX pelo apoio financeiro; à Maria de Fátima S. Lopes - Departamento de Metalurgia/ PUC-Rio pelas análises de SEM e à Professora Vera Lúcia P. Soares da Xistoquímica/IQ-UFRJ pelo uso do entalhador e equipamento de impacto.

\section{Referências Bibliográficas}

1. Bari, D. F. - Impact of Technology on Global Polyethylene in Materials, Properties, Processing \& Markets, Design Library, New York (1998).

2. Ferro, S. - Plástico Moderno, nº 304, 16 (1999).

3. Montaga, A. A. - Chemitech, 25, 44 (1995).

4. Montaga, A. A. - Hydrocarbon Processing, 57 (1994).

5. Tung, j. F.; Liew, K. F. - Proceedings of SPE ANTEC95, 962 (1995).

6. Silva, A. L. N.; Rocha, M. C. G.; Coutinho, F. M. B.; Bretas, R.; Scuracchio, C. -, J. Appl. Polym. Sci., 75, 692 (2002).

7. Guimarães, M. J. O. C.; Coutinho, F. M. B.; Rocha, M. C. G.; Garcia, M. E. F. - J. Appl. Polym. Sci., 81, 1991 (2001).

8. Kitao, K. - Polym. Eng. Sci., 37, 777 (1997).

9. Barteczak, Z.; Argon, A. S.; Cohen, R. E.; Weinberg, M. - Polymer, 40, 2331 (1999).

10. Jiang, W.; An, L.; Jiang, B. - Polymer, 42, 4777 (2001).

11. Lotti, C.; Correa, C. A.; Canevarolo, S. V. - Mat. Res., 3,37 (2000).

12. Kim, G. M.; Michler, G. H.; Gahleitner, M.; Mülhaup, R. - Polym. Adv. Techn., 9, 709 (1998).

13. Liung, H.; Jiang, W.; Zhang, J.; Jiang, B. - J. Appl. Polym. Sci., 59, 505 (1996).

14. Moore, E. P. - Principles of rubber toughening in Polypropylene Handbook, Hanser Publishers, New York, (1996).

15. Cho, K.; Yang, J.; Park, C. E. - Polymer, 39, 3073 (1998).

16. Li, W. D.; Li, R. K. Y.; Tyjong, S. C. - Polym. Test, 16, 563 (1997). 
17. Van der Wal, A.; Gaymans, R. J. - Polymer, 40, 6045 (1999).

18. Van der Wal, A.; Verheul, A. J. J.; Gaymans, R. J. Polymer, 40, 6057 (1999).

19. Wu, S. - J. Appl. Polym. Sci., 35, 549 (1988).

20. Gugumus, F.; Lelli, N. - Polym. Degrad. Stab., 72, 407 (2001).

21. Shishesag, M. R.; Donatelli, A. A. - Polym. Eng. Sci., 21, 869 (1981).

22. Yousefi, A. A.; Ait-Kadi, A.; Roy, C. - Adv. Polym. Techn., 17, 127 (1998).

23. Van der Wal, A.; Nijhof, R.; Gaymans, R. J. - Polymer, 40, 6031 (1999).
24. Willemse, R. C.; Speijer, A.; Langeraar, A. E.; Posthuma, B. - Polymer, 40, 6645 (1999).

25. Flaris, V.; Stachursky, Z. N. - J. Appl. Polym. Sci., 45, 1789 (1992).

26. Willemse, R. C.; Ramaker, E. J. J.; Van Dam, J.; Boer, A. P. - Polym . Eng. Sci., 40, 6651 (1999).

27. Willemse, R. C. - Polymer, 40, 2175 (1999).

28. Guimarães, M. J. O. C.; Rocha, M. C. G.; Coutinho, F. M. B.; Farah, M.; Bretas, R. E. S. - J. Appl. Polym. Sci., 2002 (em publicação).

Recebido: $18 / 10 / 01$

Aprovado: 15/04/02 